T.N.T., where the small crystals are irregularly disposed, this factor would not arise. But in a cast charge in which the crystal growth was carefully controlled, there might be detectable differences depending upon the orientation of the crystals with respect to the direction of propagation of the detonation wave.

Explosives Section,

$$
\text { W. C. F. SHEPHERD }
$$

Safety in Mines Research Station, Buxton. April 17.

s: Weibull, Nature, 159, 402 (1947).

'Stettbacher, "Die Schiess- und Sprengstoffe" (1933).

\section{Structure of Retained Austenite in Plain Carbon Steels}

THERE are many indications that retained austenite în quenched plain carbon steels should be regarded as a face-centred tetragonally distorted structure and not face-centred cubic as heretofore.

The tetragonality is shown either by pronounced broadening or distinct separation of the lines, depending on the carbon content in solution, that is, the amount of austenite. This is shown in the accompanying intensity curves of lines 200, 220 and 311 (photometer curves with uncorrected intensity and background equal to zero) of retained austenite present in quenched samples of steels with $0.75,0.89$ and $1 \cdot 20$ per cent of carbon. The shapes of these curves show that the tetragonality decreases with the increase of the amount of retained austenite.

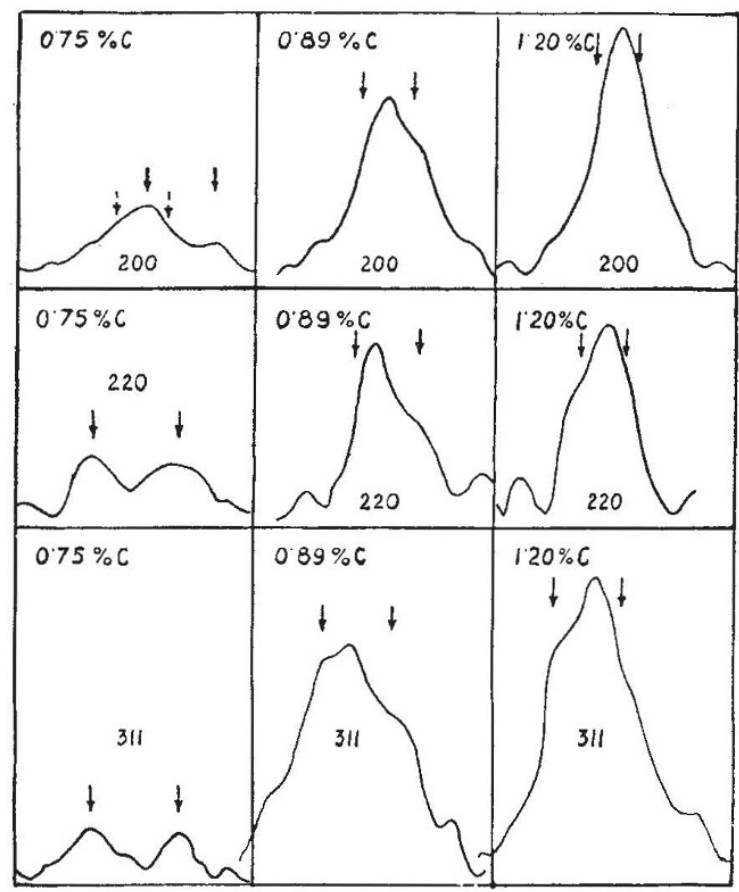

By quenching iron-carbon alloys from the gamma modification the amount of the austenitic pseudophases, which have been called $\gamma G, \gamma S$, and $\gamma E^{1}$, can be revealed. Since the grain in the above-mentioned quenched steels is made up of martensite and austenite, and since $\gamma S$ produces martensite and $\gamma E$ austenite, there is a strong 'linkage' between the two phases due to the sharing of iron atoms in the interfaces by both the austenite and the martensite.

The presence of martensite and austenite in phases differing in structure and parameter and linked closely together causes a tetragonal distortion of each with respect to the other. Martensite is tetragonally distorted in such a way that $c / a>1$, whereas in distorted austenite $c / a<1$. In the above-mentioned steels $(0.75,0.89$ and 1.20 per cent of carbon), the tetragonality of retained austenite could as a first approximation be estimated as $0.9945,0.9960$ and 0.9975 respectively.

Further investigation is in progress, and it is hoped that more detailed discussion of some of the questions which emerge from the above finding will be published in due course.

Metallurgy Department,

W. J. WrazeJ

Imperial College of Science and Technology, London, S.W.7.

1 Wrazej, W. J., Nature, 158, 308 (1946).

\section{Flow of Smoke through a Hole in Resonant Cavity}

Prof. G. D. West ${ }^{1}$ has used a smoke-filled cavity as a resonator and noted the stream of smoke issuing from a hole near the base; radiation pressure was suggested as a possible cause. In an alternative explanation ${ }^{2}$, Prof. E. N. da C. Andrade suggests that air is simply expelled during one half-cycle in a definite smoke-laden stream, and is drawn in during the next half-cycle, air being sucked in from all sides.

The matter can readily be settled by reversing Prof. West's experiment. According to Prof. Andrade's explanation, smoke outside the resonator should appear to stream in just as readily as it streamed out in the original experiment. This has been done, and the effect has been found to be reversible.

A variation of Prof. West's experiment was made as follows: A length of 2 in. diameter transparent 'Plexiglass' tubing was made to resonate as a 'closed pipe' at its first overtone (the length of the pipe thus being three quarters of the fundamental wavelength). The system was driven by a moving-coil speaker mounted at the open end of the pipe and connected to an electronic oscillator operating at 500 cycles. Pinholes were drilled in the pipe at the two pressure antinodes and at the pressure node between them. When the tube was filled with smoke and the driving source switched on, it was observed that smoke rushed from the pinholes at the antinodes but did not issue from the node if this pinhole was carefully located.

The reverse experiment was then carried out. The tube was cleared of smoke and was made a resonator as before. A short piece of similar tubing was filled. with smoke and fitted tightly to the first tube over each of the pinholes in turn, using 'Plasticine' to seal the connexion. Smoke was observed to rush into the resonating tube at both pressure antinodes.

National Research Council,

F. W. Simpson

Ottawa.

March 13.

1 Nature, 158, 755 (1946).

Nature, 159, 132 (1947). 